\title{
EVALUACIÓN PRELIMINAR DEL EFECTO DE LOS EXTRACTOS ETANÓLICOS DE CINCO PLANTAS MEDICINALES SOBRE LA MOSCA DE LOS CUERNOS Hematobia irritans L. (DIPTERA: MUSCIDAE)
}

\section{PRELIMINARY SCREENING OF ETHANOLIC EXTRACTS OF FIVE MEDICINAL PLANTS AGAINST Haematobia irritans L. (DIPTERA: MUSCIDAE)}

\author{
Mauricio Ramírez A. ${ }^{1}$ \\ Anastasia Cruz Carrillo² \\ Carlos Rodríguez Molano ${ }^{3}$
}

\section{RESUMEN}

El uso de plantas medicinales hace parte tanto de la historia de la medicina en todas las culturas del mundo como de las costumbres populares, convirtiéndose en el punto de partida para el desarrollo de medicamentos tradicionales. En respuesta a los altos niveles de resistencia y a la presencia de residuos de plaguicidas en el ecosistema, se ha buscado en las plantas nuevas alternativas para el control de insectos. A partir del conocimiento popular de algunas plantas reconocidas por su acción insecticida, con esta investigación, se buscó determinar, experimentalmente, si Nicotiana tabacum, Brugmansia arborea, Sambucus nigra, Bidens pilosa y Ambrosia cumanenses tienen efecto contra los adulta de la mosca de los cuernos, Haematobia irritans, plaga de importancia que afecta el ganado vacuno. Utilizando el método de lixiviación en frío, se preparó el extracto de las hojas de cada una de las plantas. La evaluación del efecto insecticida, se realizó in Vitro, haciendo una aspersión topical de los extractos sobre las moscas.

1 MVZ, UPTC, maomvzsi@yahoo.es

${ }^{2}$ MV, Esp UN, Docente UPTC, Programa de MVZ. Coordinador grupo GICIVET. Dirección para correspondencia: anicata22@hotmail.com

3 Z, UN Esp UPTC. Docente UPTC, Programa MVZ Coordinador grupo GIBNA. ceromol@gmail.com
Todas las plantas mostraron efecto insecticida, aunque en diferentes grados de intensidad, siendo la más efectiva la $N$ tabacum. Con estos resultados, se sustenta el conocimiento popular y se resalta la necesidad de utilizar altas concentraciones del extracto para obtener efecto insecticida.

Palabras clave: Sauco, tabaco, almatisa, borrachero, chipaca, mosca de la paleta, plaga ganado vacuno.

\section{SUMMARY}

The use of medicinal plants forms part of the medical history in every world culture, as well as of the popular customs, being also the starting point of traditional drugs. Responding to the high levels of resistance to chemical pesticides and the presence of their residues in the ecosystem, in plants, new alternatives for insect control have been searched. Based on the popular knowledge about some plants, recognized for their insecticidal action, with this research it was proposed to determine experimentally if Nicotiana tabacum, Brugmansia arborea, Sambucus nigra, Bidens pilosa and Ambrosia cumanenses have some action against and the fly, Haematobia irritans, cattle pest of importance. Leaf extracts were prepared by lixiviation in cold and the insecticidal effect was evaluated in vitro making topical aspersion of the extracts on the flies. All plants show insecticidal effect but at different intensity levels. The extract of $N$. tabacum was the most effective one. 
With these results the popular knowledge is supported, however the need of using high concentrations of the extract for obtaining insecticide effect is pointed out.

Key words: Elder, tobacco, almatisa, datura, chipaca, horn fly, cattle pest.

\section{INTRODUCCIÓN}

La mosca Haematobia irritans, conocida como "mosca de los cuernos" o "de la paleta", es un díptero hematófago que impacta negativamente la salud y la producción bovina. Dentro de los efectos adversos producidos, se destaca la transmisión de enfermedades y de otros organismos de los cuales es vector, como el caso de la transmisión de larvas de Dermatobia hominis, del Anaplasma marginale y del virus de la leucosis bovina; así mismo, induce anemia, daño de la piel, estrés, poca ganancia de peso por bajo consumo de alimento y mala conversión de alimentos. Se ha calculado que una infestación de 1000 moscas en un animal ocasiona pérdidas de $0,14 \mathrm{~g}$ de carne por mosca presente al día, conduciendo a reducciones de 8 hasta $22 \%$ del peso corporal y disminución de la ganancia de peso de $14 \%$ en novillos, efectos que traducen en pérdidas económicas considerables (Torres et al. 2008; Alonso-Díaz et al. 2007; Yepes, 2007; Leite et al. 1998).

De manera similar como se realiza para controlar otros ectoparásitos, el manejo de la mosca, entre otras acciones, incluye el uso de piretroides, organofosforados, carbamatos, así como fenilpirazol, fipronil y las lactonas macrocíclicas. Sin embargo, el empleo indiscriminado de estos fármacos generó el desarrollo de resistencia por parte del insecto, así como efectos indeseados en el medio ambiente (Suárez et al. 2006; Quijada et al. 2002; Flores et al. 2001). En respuesta a esta problemática y atendiendo a los resultados obtenidos en estudios de etnobotánica y a la medicina popular, se buscan alternativas en los extractos de plantas medicinales, para el control de parásitos externos o para el manejo de diferentes patologías, de acuerdo con los componentes que éstas tengan (De Geyter et al. 2007; López, 2002).

De acuerdo con los registros encontrados en Colombia, se destacan dentro de las plantas con efecto insecticida, la Azadirachta indica (Meliaceae), contra garrapatas; la Mammea americana (Clusiaceae), contra mosquitos; la Cyperus prolyxus (Cyperaceae); la Bidens pilosa (Asteraceae), cuya acción insecticida se ha demostrado sobre el gorgojo del maíz y sobre estados adultos y larvariaos de algunos dípteros (Iannacone et al. 2005; Gruben, 2004). La Brugmansia arbórea (Solanaceae), a pesar que en la zona del Pantano de Vargas, Boyacá, se reconoce como insecticida, la literatura la destaca por su capacidad de actuar sobre los receptores serotoninérgicos, dopaminérgicos y noradrenérgicos, a nivel de sistema nervioso central, constituyendo una planta sicoactiva aunque con algunos efectos indeseados (Capasso E Vicenzo, 2007; Gil et al. 2006; Kvist \& Moraes, 2006). Así mismo, se incluyen, como promisorios, a Sambucus nigra, Nicotiana tabacum, Borago officinalis, Equisetum bogotense, Salvia officinalis, Poligonum spp., Urtica dioca y Ruta graveolens (Edafón, 2006; Gruben, 2004).

En el 2007, los grupos de investigación GICIVET y GIBNA, pertenecientes al Programa de MVZ de la Universidad Pedagógica y Tecnológica de Colombia, desarrollaron un trabajo que permitió conocer cuáles eran las plantas de uso medicinales que la población reconocía y utilizaba con mayor frecuencia en la región de Paipa, Boyacá (Cruz E Rodríguez, 2007). A partir de estos resultados, se seleccionaron las cinco plantas de hallazgo más comunes en la zona y que son utilizadas por sus habitantes, para el control de parásitos externos, moscas y pulgas, principalmente, sin importar si estas plantas fueran o no nativas de la región. Partiendo de lo anterior, el propósito de este estudio fue evaluar la eficacia in Vitro de los extractos etanólicos de Nicotiana tabacum, Brugmasia arborea, Sambucus nigra, Bidens pilosa y Ambrosia cumanenses de ocurrencia común en el departamento de Boyacá, contra la mosca de los cuernos H. irritans (Diptera: Muscidae).

\section{MATERIALES Y MÉTODOS}

El estudio fue de tipo experimental in Vitro y se desarrolló en tres fases: la primera consistió en hacer la colecta de las plantas en los municipios de Tunja y de Rondón, en el departamento de Boyacá, Colombia, así como la elaboración de los extractos, que se llevó a cabo en el Laboratorio de Control Biológico de la Facultad de Ciencias Agropecuarias, de la Universidad Pedagógica y Tecnológica de Colombia (UPTC), sede Tunja; la segunda fase, se relacionó con la captura de las moscas en el municipio de Tunja y el mantenimiento de las mismas, 
en un espacio adaptado para tal fin, en el laboratorio de Nutrición Animal del Programa de Medicina VeterinariaZootecnia de la UPTC y la tercera fase, consistió en evaluar el efecto de los extractos y de sus diluciones, sobre adultos de la $H$. irritans.

Colecta de las plantas y elaboración de los extractos. Se seleccionadas las cinco plantas más reconocidas por los habitantes del Pantano de Vargas, como plantas con efectos insecticidas y fueron ellas, Ambrosia cumanenses (altamisa), B. pilosa (chipaca), B. arborea (borrachero), S. nigra (sauco) y N. tabacum (tabaco). En todos los casos, las hojas se cortaron con tijeras en cantidad superior a $200 \mathrm{~g}$ y se transportaron en sobres de manila para evitar la humedad, hasta el laboratorio, donde se dispusieron en una superficie plana, separadas unas de otras para realizar el secado, a temperatura ambiente, en un lugar cubierto y seco.

El método escogido para la obtención de los extractos de cada una de las plantas analizadas fue el de lixiviación en frío (Sharapin, 2000), utilizado como método de extracción de metabolitos de las plantas medicinales, que permite extraer todos los componentes liposolubles afines a solventes alcohólicos. Para ello, se empleó una columna de extracción de PVC con mangueras conectadas, conformando un circuito cerrado, que permitió re-utilizar el solvente. Fue así como de cada planta se tomaron $200 \mathrm{~g}$ de hojas secas maceradas y se colocaron en la columna, empacando las dos terceras partes de su capacidad. Posteriormente, se llenó la columna con una solución de etanol $20 \%$ y se conectó el motor para mantener constante el flujo del solvente, durante 24 horas.

Cumplido el tiempo, una de las mangueras se desconectó y el contenido eluido fue recogido en un Erlenmeyer; a continuación, se adicionó más solvente, repitiendo el procedimiento varias veces, hasta que el solvente saliera traslúcido. Cada proceso culminó con la evaporación del solvente del respectivo extracto recogido, mediante un baño maría, obteniendo así, el extracto bruto $100 \%$, el cual, se conservó a 4oC (Davicino et al. 2007; Edafón, 2006; Lock et al. 2006; Ferro E de Alvarenga, 2000). Todos los extractos fueron evaluados por observación directa para determinar características de olor, de color, de consistencia y de volumen obtenido.

El extracto, así preparado, sirvió para elaborar las diluciones correspondientes utilizando agua destilada.
Finalmente y en búsqueda de corroborar la presencia de compuestos químicos en cada uno de los extractos puros obtenidos, se realizó una cromatografía en capa fina con lámina de sílica, para observar los compuestos eluidos, por fluorescencia.

Captura y mantenimiento de las moscas. Las moscas, se capturaron con una red entomológica, a partir de bovinos infestados con éstas, en las instalaciones de la UPTC y se ubicaron en jaulas de 30x30x30cm, elaboradas para el estudio con balso y tela mosquitera. Una vez transportadas al laboratorio de Parasitología para su identificación, fueron divididas en grupos de diez y ubicadas en las jaulas sobre los mesones del laboratorio, bajo iguales condiciones medioambientales. Para la alimentación de las moscas, se dispuso diariamente, en cada una de las jaulas, una toalla sanitaria impregnada en sangre fresca de bovino con citrato, como anticoagulante y kanamicina, para evitar la contaminación bacteriana. En todos los casos, se comprobó por observación directa que las moscas se alimentaban (Gallardo, 2000).

Evaluación del efecto insecticida de los extractos diluidos. Para la evaluación de cada uno de los extractos así como de las diluciones preparadas a partir de éstos, se utilizaron dos grupos de moscas de diez individuos cada uno, constituyendo así, el grupo tratado y su réplica.

A pesar que en el planteamiento inicial del estudio se propuso la evaluación del efecto insecticida de los extractos puros a través de la aspersión de los mismos sobre las moscas fue imposible realizarlo debido a su viscosidad y, por ello, en todos los casos, se inició con una dilución 5:10 en agua destilada. La aplicación de los extractos, se hizo por aspersión utilizando un atomizador comercial del mismo calibre, para todos los grupos, aplicando un disparo completo, para cada grupo de diez moscas.

Las diluciones preparadas no fueron necesariamente las mismas para todos los extractos, puesto que en este trabajo, se aplicó el método de máximas y mínimas, empleado en farmacología, para hallar dosis efectivas de moléculas nuevas. Bajo esta premisa, la concentración de los extractos se aumentó o disminuyó, aproximadamente $50 \%$ respecto a la anterior, de acuerdo con los resultados obtenidos. Así, cuando hubo mortalidad de las moscas, se preparó el extracto en mayor dilución hasta encontrar 
la concentración mínima eficaz y al no observar efecto, se trabajó con preparaciones más concentradas, hasta encontrar la que mostrara eficacia.

Con el fin de dar mayor confiabilidad a los resultados, se trabajó un grupo control positivo, el cual, se enfrentó a un insecticida piretroide comercial a base de cipermetrina, al $15 \%$, preparado según las indicaciones de la etiqueta (dilución 1:1000 en agua corriente); se asperjó sobre las moscas, de la misma forma como se efectuó con las diluciones de los extractos. Igualmente, se dispuso de un grupo control, donde las moscas fueron asperjadas con agua destilada de la misma procedencia de la que se utilizó para preparar las diluciones. Durante todo el estudio, se mantuvo un grupo de moscas (control negativo), que no estuvo expuesto a ninguna sustancia insecticida, con el fin de garantizar que las condiciones medioambientales del laboratorio eran adecuadas, para el mantenimiento de las moscas.

La mortalidad de las moscas, se determinó por ausencia de movimiento de las patas y las alas, al ser tocadas una a una con un objeto de punta roma. Este procedimiento, se efectuó al cabo de 24 horas de la exposición a cada uno de los tratamientos, tanto en los grupos tratados como en las réplicas. Se consideró como valor mínimo de eficacia insecticida, una mortalidad de $60 \%$, por lo que, valores por debajo de éste se interpretaron como ineficacia (Gallardo, 2000). Los porcentajes de mortalidad, se compararon entre grupos para cada una de las diluciones y se determinó el grado de eficacia, a través del programa EPIDAT, para el análisis epidemiológico de datos tabulados versión 3.0.

\section{RESULTADOS Y DISCUSIÓN}

Con el método de extracción, se obtuvieron los extractos que mostraron similares características de color, volumen obtenido y consistencia (Tabla 1). Para todos los tratamiento valorados, se observó el mismo comportamiento entre el grupo tratado $(n=10)$ y su réplica $(n=10)$, sin diferencias significativas entre ambos, por lo que los resultados porcentuales corresponden al promedio obtenido entre ambos, es decir, a 20 moscas expuestas a cada uno de los tratamientos.

A. cumanenses (altamisa, artemisa, ajenjo, madra, tomajaras). De acuerdo con los resultados obtenidos en este trabajo, para esta planta, se evaluaron cuatro diluciones, 5:10, 2,5:10, 1,2:10 y 0,6:10. Con la primera, se obtuvo $100 \%$ de mortalidad de las moscas; con la segunda, tercera y cuarta dilución las mortalidades fueron de 65,60 y $10 \%$, respectivamente, por lo cual, con concentraciones inferiores a 1,2:10, se pierde la eficacia del extracto de esta planta contra la mosca $H$. irritans.

La altamisa, a pesar de ser una planta originaria de regiones templadas de Europa, fue introducida a América y se adaptó fácilmente en diferentes tipos de suelos en Colombia (Jiménez et al. 2005; Bermúdez E Velásquez, 2002). Los estudios de etnobotánica desarrollados en América Latina reportan múltiples usos, dentro de los que se destaca su utilización como analgésico, antiinflamatorio, antipirético, para el manejo de cefalea, hipertensión y estreñimiento y, con menor frecuencia, se encuentran reportes de su efecto

Tabla 1. Características físicas de las plantas evaluadas.

\begin{tabular}{|l|c|c|c|c|}
\hline \multicolumn{1}{|c|}{ Nombre Científico } & $\begin{array}{c}\text { Volumen } \\
\text { Obtenido }(\mathrm{ml})\end{array}$ & Color & Olor & Consistencia \\
\hline Ambrosia cumanenses & 65 & Carmelito & Característico a la planta & Viscoso \\
\hline Bidens pilosa & 70 & Carmelito & Característico a la planta & Viscoso \\
\hline Sambucus nigra & 50 & Carmelito & Característico a la planta & Viscoso \\
\hline Nicotiana tabacum & 45 & Carmelito inteso & Característico a la planta & Muy viscoso \\
\hline Brugmasia arborea & 40 & Verde & Dulzón & Viscoso \\
\hline
\end{tabular}


antiparasitario, contra nematodos y moscas (Toscano, 2006; Bermúdez E Velásquez, 2002; León et al. 2002; Peguero, 2002). Dentro de los componentes reportados, se destaca el felandreno, el acetato de linalilo y el ocimeno, alcaloides presentes en las hojas, con acción antihelmíntica y repelente demostradas (Carrillo-Rosario $\varepsilon$ Díaz, 2005); en este estudio, lo que se observó fue acción insecticida en corto tiempo. El efecto disminuyó de manera proporcional a la concentración del extracto, perdiendo eficacia en altas diluciones. Considerando la ausencia de literatura científica que indique el efecto aquí encontrado, este sería el primer reporte de la acción insecticida de la $A$. cumanenses que valdría la pena seguir estudiado, para determinar cuál de los alcaloides que posee, le confiere esta característica.

B. pilosa (chipaca, romerillo blanco, amor seco, cadillo rosero, margarita silvestre). El extracto de chipaca fue evaluado en cuatro diluciones; se inició con la dilución 5:10, que no mostró eficacia, por lo que se elaboraron dos diluciones de mayor concentración, 6,2:10 y 7,5:10 (Tabla 1). La chipaca es una hierba anual distribuida, principalmente, en regiones tropicales y subtropicales de todo el mundo, cuyos análisis fitoquímicos indican que sus principales componentes activos se encuentran en los aceites obtenidos de las hojas y flores (Deba et al. 2008). A pesar que la medicina popular reconoce esta planta como medicinal para el tratamiento de aterosclerosis, malaria, hepatitis, infecciones urinarias e infecciones causada por herpes, virus de las sustancias químicas que posee (taninos, esteroles, esteroides, flavonoides, glicósidos aurona y chalconas y polisacáridos, entre otros) y de la gran variedad de efectos medicinales, demostrados científicamente (antineoplásico, antioxidante, cicatrizante, antipirético, antibacteriano y antifúngico) (Deba et al. 2008; Arroyo et al. 2007; Sudararajan et al. 2006; Chiang et al. 2003; Lastra E Ponce De León, 2001), no se encuentran reportes que soporten la acción insecticida observada en el presente estudio, excepto por los estudios realizados sobre el gorgojo del maíz (Iannacone et al. 2005). Sin tener un soporte científico, se presume que los principios insecticidas que esta planta pueda contener, corresponden al grupo químico de los alcaloides, que se destacan como compuestos naturales con acción insecticida (Ferrero et al. 2004).

De acuerdo con los resultados hallados y comparando la chipaca con la altamisa, se puede indicar que la acción insecticida de esta última es más notoria, en razón a que mantuvo su eficacia en diluciones superiores a la lograda con la chipaca.

B. arborea (borrachero, floripondios). En este estudio y de igual forma que la altamisa, el borrachero se evaluó en cuatro diluciones $5: 10,2,5: 10,1,2: 10$ y 0,6:10 mostrando efecto satisfactorio contra la $H$. irritans hasta la dilución 1,2:10, a partir de la cual, ésta se perdió (Tabla 2).

Tabla 2. Mortalidad producida por diferentes diluciones de extracto etanólico de tres plantas medicinales en la mosca de los cuernos Haematobia irritans.

\begin{tabular}{|c|c|c|c|}
\hline & Bidens pilosa & Brugmasia arborea & Nicotiana tabacum \\
\hline Dilución & \multicolumn{3}{|c|}{$\%$ Mortalidad } \\
\hline $7,5: 10$ & 100 & - & - \\
\hline $6,2: 10$ & 95 & - & - \\
\hline $5: 10$ & 55 & 100 & 100 \\
\hline $2,5: 10$ & 25 & 100 & 100 \\
\hline $1,2: 10$ & - & 75 & 100 \\
\hline 0,6:10 & - & 20 & 100 \\
\hline 0,3:10 & - & - & 100 \\
\hline $0,15: 10$ & - & - & 100 \\
\hline $0,1: 10$ & - & - & 90 \\
\hline $0,05: 10$ & - & - & 65 \\
\hline
\end{tabular}


Posiblemente, el borrachero es más conocido por sus efectos alucinógenos y tóxicos que medicinales, por la presencia de ciertas sustancias como la escopolamina, la hioscina y la atropina (Arango, 2008; Kvist E Moraes, 2006). A pesar que esta planta es reconocida popularmente en Boyacá y en otras regiones del país por sus efectos insecticidas, no se encuentran estudios científicos que demuestren esta acción. Bajo esas condiciones, la presente investigación constituye una de las primeras demostraciones que respaldan el conocimiento popular que indica el uso de esta planta como insecticida. Por otra parte y conociendo la forma de actuar de sus componentes, se puede inferir que la acción insecticida se genera por parálisis de la mosca, debida a la acción anticolinérgica ejercida por el bloqueo de los receptores colinérgicos en la placa neuromotora del insecto (Arango, 2008).

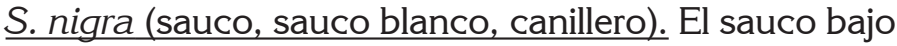
las condiciones planteadas en este estudio, mostró efecto insecticida en diferentes diluciones. Fue así como las diluciones evaluadas 7,5:10 - 6,2:10 - 5:10 y 2,5:10 indicaron eficacia con mortalidades de $100 \%$, $95 \%, 60 \%$ y $70 \%$, respectivamente. En dilución 1,2:10 el resultado obtenido, se interpretó como ineficacia, con mortalidades por debajo de $60 \%$. A pesar que en todos los ensayos realizados para cada planta los extractos más concentrados mostraron mayor eficacia insecticida, en este grupo, la dilución 2,5:10 fue más eficaz que la dilución 5:10, lo cual, no concuerda con la relación directa, concentración eficacia, que se observó a lo largo del estudio. Otra posible explicación es que los extractos de alta viscosidad difícilmente pasan por el atomizador dificultando la dispersión; sin embargo, ello no ocurrió con las formas más concentradas que tuvieron mayor viscosidad. Sin tener otra explicación y considerando que el agua en que se preparó la dilución fue la misma así como las condiciones medioambientales, se asume que este resultado fue producto de la individualidad propia de los organismos vivos.

Esta planta de distribución mundial, se encuentra con frecuencia en el departamento de Boyacá (Cruz $\mathcal{E}$ Rodríguez, 2007). Los efectos hallados, se justifican por la presencia de gran cantidad de compuestos, dentro de los que se destacan flavonoides, aceites cafeíco y clorofénicos, ácidos ursólico y oleanólico, esteroles, glucósidos, alcaloides, triterpenos y taninos, distribuidos en diferentes partes, por lo que, a nivel de medicina popular, se usan con diferentes fines. Aunque en este estudio el efecto observado fue de tipo insecticida, la literatura resalta la utilización del follaje como repelente de moscas en equinos, debido al intenso olor que libera. De manera similar a como sucede con otras plantas de uso por tradición cultural, se encuentran pocos hallazgos científicos que respaldan esas creencias; aún así, algunos autores demuestran efecto antioxidante, diurético, purgante y hemostático. Así mismo, se emplea en el tratamiento de diabetes, tos, bronquitis, hemorroides, fiebres e insomnio (Dawidowicz et al. 2006; Toscano, 2006; León et al. 2002; Gray et al. 2000) y, de manera similar a lo ocurrido con la chipaca, existen reportes de su eficacia contra el gorgojo del maíz, pero no contra ningún otro artrópodo (lannacone et al. 2005).

Con los resultados logrados en este estudio, se puede afirmar que el sauco tiene efecto insecticida contra la $H$. irritans, aunque no se encuentren otros reportes científicos con los que se puedan comparar estos hallazgos.

N. tabacum (tabaco). En este estudio, el extracto etanólico de tabaco fue el que mostró mayor eficacia contra la mosca de los cuernos, debido a que se observó mortalidad, en diluciones superiores a las que fueron eficaces para las otras plantas evaluadas. Para esta planta, se evaluaron las diluciones 5:10, 2,5:10, 1,2:10, 0,6:10, 1:10 y 0,5:10 (Tabla 2).

A diferencia de las especies anteriores, el tabaco ha sido reconocido, no solo a nivel popular sino también en estudios científicos, como planta con propiedades insecticida de contacto y de absorción; sin embargo, el uso sobre $H$. irritans no se había reportado. A pesar que el lapso determinado para evaluar la mortalidad en este estudio fue de 24 horas, se puede destacar que con esta planta la mortalidad ocurrió de manera casi inmediata para todas las diluciones que fueron eficaces, lo que soporta la alta efectividad observada, bajo las condiciones de este ensayo.

El tabaco posee componentes activos, como nicotina, cumarina, alontaína, pirrolidina, nicotelina, ácido nicotínico y nicotirina, que le confieren efectos narcóticos y alucinógenos, así como el efecto insecticida, demostrado en este estudio (Kvist $\varepsilon$ Moraes, 2006; Capasso \& De Feo, 2003). Partiendo de que el principal componente del tabaco es la nicotina, se asume que el 
efecto se debió a parálisis producida sobre la mosca por estímulo sostenido sobre los receptores nicotínicos de la placa motora. La eficacia de la molécula de nicotina ha conducido al desarrollo de derivados moleculares, los insecticidas neonicotinoides, que actúan de manera similar a la molécula natural y posiblemente son menos tóxicos para los mamíferos, constituyendo un nuevo grupo químico para la industria de los plaguicidas (Tomizawa E Casida, 2008).

Con relación a los resultados alcanzados con el grupo control positivo, se corrobora la eficacia del producto utilizado y del método aplicado, ya que hubo mortalidad de $100 \%$ en la dilución indicada por la etiqueta (1:1000). Con el grupo control negativo no se observó ninguna muerte así, como tampoco con las moscas que se mantuvieron en las jaulas sin ningún tipo de intervención. En consecuencia, se demuestra que la mortalidad lograda con los diferentes extractos, se debió a la acción insecticida de los mismos y no al agua utilizada para la dilución, a condiciones medioambientales o a la falta de alimento.

\section{CONCLUSIONES}

Todas las plantas aquí evaluadas mostraron, en algún grado, efecto insecticida, destacándose dentro de todas la $N$. tabacum. que mantuvo su efecto aún en bajas concentraciones. En todos los casos, se evidenció que la eficacia del extracto disminuye de manera progresiva, a medida que se aumenta la dilución del mismo, situación que se explica por la relación dosis-respuesta, que se observa en la mayoría de sustancias farmacológicamente activas (Goodman E Gilman, 2006). De manera complementaria y en razón a que las moléculas biológicamente activas producen efectos deseados, en ciertas dosis también pueden desencadenar reacciones adversas, que para el caso de los insecticidas de síntesis, han sido notorias en animales y en humanos, lo que hace necesario determinar no solo la eficacia de los productos naturales sino el grado de seguridad que ofrecen al ser aplicados en los animales.

\section{RECOMENDACIONES}

Teniendo en cuenta que los componentes activos presentes en las plantas varían por múltiples factores, se recomienda desarrollar trabajos similares, incluyendo algunas variables, como diferentes partes de la planta, extracción acuosa, extracción alcohólica usando otros solventes y extracción en caliente, para finalmente llegar a identificar los principios activos con acción insecticida. Así mismo, una vez se demuestre in Vitro el efecto insecticida, se recomienda hacer valoraciones in Vivo para determinar su eficacia en los animales y estudios de toxicidad, que permitan conocer el riesgo del uso de las plantas promisorias en las especies animales de interés.

\section{AGRADECIMIENTOS}

Los autores de este trabajo expresan sus agradecimientos a los funcionarios y directivos del Laboratorio de Control Biológico de la Facultad de Ciencias Agropecuarias, de la UPTC y a la doctora Astrid Martínez por su orientación en la obtención de los extractos.

\section{BIBLIOGRAFÍA}

ALONSO-DÍAZ, M.A.; ACOSTA-RODRÍGUEZ, A.; MALDONADO-SIMÁN, E.; RAMÍREZ-VALVERDE, R., BERMÚDEZ-VILLANUEVA, L. 2007. Dinámica poblacional de Haematobia irritans en bovinos del trópico mexicano. Rev. Cient. (Maracaibo). 17(4):330-334. Disponible desde Internet en: http://www.scielo.org.ve/scielo.php (con acceso abril 10/04/08).

ARANGO, A.G.J. 2008. Alcaloides y compuestos nitrogenados. Medellín, Colombia. 82p. Disponible desde Internet en: http://farmacia.udea.edu. co/ fff/alcaloides.pdf (con acceso 13/07/08).

ARROYO, J.; BONILLA, P.; RAEZ, E. 2007. Phenolic compounds from Bidens pilosa methanolic fraction on induced gastric neoplasia in rats. Ann. Fac. Med. 68(2):105-112.

BERMÚDEZ, A.; VELÁSQUIEZ, E. 2002. Etnobotánica médica de una comunidad campesina del estado de Trujillo, Venezuela: un estudio preliminar usando técnicas cuantitativas. Rev. Facultad de Farmacia. 44:2-6.

CAPASSO, A.; VICENZO, D. 2007. In vitro binding receptors study by Valeriana ascendens, Iresini herbstii and Brugmansia arborea extracts. Med. Chem. 3(6):599-604. 
CAPASSO, A; DE FEO, V. 2003. Alkaloids from Brugmansia arbórea (L.) reduce morphin withdrawal in vitro. Phytother. Res. 17(7):826829.

CARRILLO-ROSARIO, T; DÍAZ de R., A. 2005. Actividad antimalárica de extractos crudos de plantas en ratones infectados con Plamodium berghei. Rev. Facultad de Farmacia. 47(1):2-9.

CHIANG, L.C., CHANG, J.S., CHEN C.C., LIN, C.C. 2003. Antiherpes simplex virus activity of Bidens pilosa and Houttuynia cordata. Am. J. Ch. Med. 31(3):355-362

CRUZ, C.A.; RODRÍGUEZ, M.C.E. 2007. Caracterización de la Riqueza Botánica del Pantano de Vargas, Paipa (Boyacá). Informe final de trabajo de investigación. Disponible en Biblioteca INIAG, UPTC-Tunja. 63p.

DAVICINO, R.; MATTAR, M.A.; CASALI, Y.A.; CORREA, S.G.; PETTENATI, E.M.; MICALIZZI, B. 2007. Actividad antifúngica de extractos de plantas usadas en medicina popular en Argentina. Rev. Peru Biol. 14 (2): 247-252

DAWIDOWICZ, A.L.; WIANOWSKA, D.; BARANIAK, B. 2006. The antioxidant properties of alcoholic extracts from Sambucus nigra L. (antioxidant properties of extracts). Lebensmittel - Wissenschaft + Technologie. 39(3):308-315.

DEBA, F.; TRANG, X.T.; YASUDA, M.; TAWATA, S. 2008. Chemical composition and antioxidant, antibacterial and antifungal activities of the essential oils from Bidens pilosa Linn. var. Radiata. Food Control. 19(4):346-352.

DE GEYTER, E.G; SMAAGGHE, G. 2007. First results on the insecticidal action of saponins. Commun Agric Appl Biol Sci. 72(3):645-648.

EDAFÓN, Fundación Agroecológica. 2006. Plantas para el control de ectoparásitos. Disponible desde Internet en: http://www.controlbiologico. com/index.htm (con acceso 03/08).

FERRO, E.; DE ALVARENGA, N.C. 2000. Evaluación de la actividad antimicrobiana de Aristolochi giberti.
Revista de Ciencia y Tecnología (Universidad Nacional de Asunción, Paraguay). 1(2):71-77.

FERRERO, A.A.; DESCAMPS, L; REVIRIEGO, M. 2004. Biotecnología y Mejoramiento Vegetal. Cap. 10 "Estrategias para el control de insectos plaga" p.335-342. Disponible desde Internet en: http:// www.inta.gov.ar/ediciones/2004/biotec/parte8_ cap10.pdf (con acceso 12/12/08).

FLORES, A.E.; BADII. M.H.; PONCE, G. 2001. Resistencia a insecticidas en insectos vectores de enfermedades con énfasis en mosquitos. Rev. Salud Pública y Nutrición. 2(4):34-37.

GALLARDO, J. 2000. Mortalidad de la mosca de cuerno Haematobia irritans causada por el pigmento Floxin B.. Tec Pecuaria, Méx. 38:211-217. Disponible desde Internet en: www.tecnicapecuaria. org.mx (con acceso 25/03/08).

GOODMAN, G. 2006. Las Bases Farmacológicas de la Terapéutica. 10 ed. Vol.1. McGraw. México.

GIL, O.R.; CARMONA, A.J., RODRÍGUEZ, A.M.C. 2006. Estudio etnobotánico de especies tóxicas, ornamentales y medicinales de uso popular, presentes en el Jardín de Plantas Medicinales "Dr. Luis Ruiz Terán" de la Facultad de Farmacia y Bioanálisis de la Universidad de Los Andes. Boletín Antropológico. 24(68):463-481.

GRAY, A.M.; ABDEL-WAHAB, Y.H.A.; FLATT, F.R. 2000. The traditional plant treatment, Sambucus nigra (elder), exhbits insulin-like and insulin releasing actions in vitro. J. of Nutrition. 130:15-20.

GRUBEN, G.J. 2004. Plant Resources of Tropical Africa. Backhuys Publishiers, O. A. Denton. Publicado por Prota 2. p.114-117. Disponible desde Internet en: http://books.google.com.co (con acceso 23/05/08).

IANNACONE, J.; AYALA H.; AMID, R. 2005. efectos toxicológicos de cuatro plantas sobre el gorgojo del maiz Sitophilus zeamais Motschulsky 1855 (Coleoptera: Curculionidae) y sobre el gorgojo de las galletas Stegobium paniceum (Linnaeus 1761) (Coleoptera: Anobiidae) en Perú. Gayana. 69(2):234-240. 
JIMÉNEZ, O.A.M.; RAMOS, P.Y.A.; GARCÍA, C.F.; RÍOS, H.A.; ASPRILLA, P.J. 2005. El Chocó una fuente de oportunidades comerciales, a partir del conocimiento, valoración y manejo de su biodiversidad. Rev. Inst. U. Tecnológica del Chocó. 22:3-9.

KVIST, L.P.; MORAES, R.M. 2006. Plantas sicoactivas. Botánica Económica de los Andes Centrales. 12:294-312.

LASTRA, V.H.A.; PONCE DE LEÓN, R.H. 2001. Bidens pilosa Linné. Rev. Cubana de Plantas Medicinales. 6(1):28-33.

LEITE, R.C.; RODRÍGUEZ, Z.; FACCINI, J.L.H.; OLIVEIRA, P.R.; FERNÁNDES, A.A. 1998. First Report of Haematobia irritans (L.) (Diptera: Muscidae) as vector of Dermatobia hominis (L.jr.) (Diptera: Cuterebridae) in Minas Gerais, Mem. Inst. Oswaldo Cruz. 93:761-762.

LEÓN, H.J.; VALERO, H.; GIL, O.R. 2002. 23 Especies vegetales medicinales de uso frecuente en la población de Tabay. Rev. Facultad Farmacia 44:51-58.

LÓPEZ, L.M.T. 2002. Formas de administración más habituales de plantas medicinales. Fitoterapia. 21(2):122-125.

LOCK. O.; CABELLO, I.; DOROTEO, V.H. 2006. Analysis of flavonoids in plants. Universidad Católica del Perú, sección de Química. Disponible desde Internet en: www.iupac.org/publications/cd/ medicinal_chemistry (con acceso 01/07/08).

PEGUERO, B. 2002. Estudio etnobotánico de las comunidades ubicadas dentro y en la periferia del Parque Nacional Juan Bautista Pérez Rancier. Evaluación Ecológica Integrada del Parque Nacional Juan Bautista Pérez Rancier. Disponible desde Internet en: http:/www.parksinperil.org/ files/d_1_n_iii_libro_evaluacinecolgica_ii_parte. pdf (con acceso 20/12/07).

QUIJADA, T.; MARCHÁN, V.; CARUSI, P.; JIMÉNEZ, M.; GARCÍA, M. 2002. Efecto del control químico sobre Haematobia irritans (Linaeus 1758) (Diptera: Muscidae) durante un año en bovinos de la Parroquia Moroturo, municipio Urdaneta del estado Lara, Venezuela. Rev. Científica. 12(supl. 2):601-603.

SHARAPIN, N. 2000. Fundamentos de Fitotectonología de Productos Fitoterapéuticos. Convenio Andrés Bello. 230p. Disponible desde Internet en: http:// books.google.com.co/books?id=XH2HzSIJPywC $\varepsilon p g=$ PA68Elpg $=$ PA68Edq $=$ extraccion + alcoho lica + plantas + vol.Esource $=$ webEots $=i$ RpuLYNI FqEsig =Afh268RAmTa7UdKTzJmyGsQZy0Ehl $=$ esEsa $=$ XEoi $=$ book_resultEresnum $=1 E c t=r e$ sult\#PPP1,M1 (con acceso 12/10/08).

SUÁREZ, V.H.; CASTELLI, M.E.; AGUIRRE, D.H.; CAFRUNE, M.M.; CETRÁ, B.; FADER, O.W. 2006. El uso de insecticidas para el control de Haematobia irritans (L.) (Diptera:Muscidae) en la Argentina. Rev. Invest. Agropec. 35(2):21-35.

SUNDARARAJAN, P.; DEY, A.; SMITH, A.; DOSS, A.G.; RAJJAPAN, M.; NATARAJAN, S. 2006. Studies of anticancer and antipyretic activity of Bidens pilosa whole plant. African Health Sciece. 6(1):27-30.

TOMISAWA, M; CASIDA, J.E. 2008. Molecular recognition of neonicotinoid insecticides. The determinants of died of dead. Acc Chem Res en proceso de publicación. Disponible desde Internet en: http://www.ncbi.nlm.nih.gov/ pubmed/ 19053239?ordinalpos $=2$ Eitool $=$ EntrezSystem2 . PEntrez.Pubmed.Pubmed_ResultsPanel.Pubmed_ DefaultReportPanel.Pubmed_RVDocSum (con acceso 12/12/08).

TORRES, R.L.; ALMAZARÁN, C.; ROSARIO-CRUZ, R; QUIROZ, R.H; De La FUENTE, J. 2008. Identificación DE antígenos protectores contra Haematbia irritans mediante interferencia de ARN. Memorias del I Foro Universitario "Investigación, Sociedad y Desarrollo", Avances y Perspectivas: 65-69. Disponible desde Internet en: http://www.turevista.uat.edu.mx / Volumen\%203\%20numero\%202/PRIMER\%20FO RO\%20UNIVERSITARIO/CA-13.swf (con acceso el 12/12/08). 
TOSCANO, G.J.Y. 2006. Uso tradicional de plantas medicinales en la vereda San Isidro, municipio de San José de Pare, Boyacá: Un estudio preliminar usando técnicas cuantitativas. Acta Biol. Colombiana. 11(2):137-146.
YEPES, M.L.Y. 2007. Fluctuación de Haematobia irritans L. (Diptera: Muscidae) en bovinos de clima cálido. Rev. Fac. Nal. Agronomía. 60(1):36-39.

Recibido: Agosto 4 de 2008

Aceptado: Febrero 4 de 2009 